Original Research Paper

\title{
In Silico Cholinesterase and Monoamine Oxidase Inhibitory Activities of Perillaldehyde and D-Limonene, Main Compounds of Essential Oil of Algerian Ammodaucus leucotrichus
}

\author{
${ }^{1}$ Nesrine Sadaoui-Smadhi, ${ }^{1}$ Souad Khemili-Talbi, ${ }^{2}$ Wadood Abdul, ${ }^{3}$ Souheyla Toubal, \\ ${ }^{3,4}$ Wafa Mokhtari, ${ }^{3}$ Narimen Benhabyles, ${ }^{3}$ Karim Arab, ${ }^{5}$ Khettal Bachra and ${ }^{6}$ Rahim Fazal \\ ${ }^{1}$ Departement de Biologie, Faculté des sciences, \\ Université M'hamed Bougara de Boumerdes, Avenue de l'independance, Boumerdes 35000, Algeria \\ ${ }^{2}$ Department of Biochemistry, Abdul Wali Khan University, Mardan, Khyber Pakhtunkhwa, Pakistan \\ ${ }^{3}$ Laboratoire de Valorisation et de Conservation des Ressources Biologiques (VALCORE), \\ Département de Biologie, Faculté des Sciences, Université de Boumerdes, Boumerdes, Algeria \\ ${ }^{4}$ Unité Computational Biology and Bioinformatics (service 3BIO, EPB), \\ Université Libre de Bruxelles, CP 165/61, avenue F. Roosevelt 50, 1050 Bruxelles, Belgium \\ ${ }^{5}$ Laboratoire de Biotechnologies Végétales et Ethnobotanique, \\ Faculté des Sciences de la Nature et de la Vie, Université de Bejaia, Bejaia 06000, Algeria \\ ${ }^{6}$ Department of Chemistry, Hazara University, Mansehra, Khyber Pakhtunkhwa, Pakistan
}

\author{
Article history \\ Received: 26-12-2019 \\ Revised: 11-02-2020 \\ Accepted: 26-02-2020 \\ Corresponding Author: \\ Nesrine Sadaoui-Smadhi \\ Departement de Biologie, \\ Faculté des Sciences, \\ Université M'hamed Bougara \\ de Boumerdes, Avenue de \\ l'independance, Boumerdes \\ 35000, Algeria \\ Email: n.sadaoui@univ-boumerdes.dz
}

\section{Introduction}

Alzheimer's Disease (AD) is a neurodegenerative disorder that is characterized by progressive deterioration of memory and cognition (Terry and Buccafusco, 2003). The low level of acetylcholine is the most important modification observed in the brain in Alzheimer's patients. Acetylcholine is liberated at the synaptic gap and it is a neurotransmitter which plays a crucial role in memory and cognition (Dall'Acqua et al., 2010; Lu et al., 2011). It is cleaved by the action of cholinesterase (ChE) enzymes to produce choline and acetate (Quinn, 1987; Sussman et al., 1991). There are

\begin{abstract}
In a continuation of our previous work for the exploration of novel enzyme inhibitors, molecular modeling was used to inspect the binding mode of perillaldehyde and D-limonene, the major compounds of essential oil of Algerian Ammodaucus leucotrichus into the active site pocket of cholinesterase ( $\mathrm{AChE}$ and $\mathrm{BuChE}$ ) and Monoamine Oxidase (MAO). The molecular docking was carried out using Molecular Operating Environment (MOE) software package. Docking analysis showed that this compounds (perilladehyde and D-limonene) can interact with both the Catalytic Active Site (CAS) of AChE, BuChE and MAO. For D-limonene, molecular docking BuCl compared with compound D-Limonene. The best interaction between perilladehyde and monoamine oxidase was also revealed. This paper shows best correlation between the in vitro study and the in silico molecular docking study of anti-cholinesterase and anti-monoamine oxidase activities.
\end{abstract}

Keywords: Acetylcholinesterase, Butyrylcholinesterase, Monoamine Oxidase, Molecular Docking, Perilladehyde, D-limonene 
important role in psychiatric disorders, such as depression and anxiety, whereas Monoamine oxidase-B $\mathrm{MAO}-\mathrm{B}$ is more specific to other neurotransmitters as phenylethylamine and it is involved in neurological disorders, such as Alzheimer's and Parkinson's disease (Cesura and Pletscher, 1992; Youdim, 1995). In view of the limited number of cholinesterase inhibitors currently available for the treatment of $\mathrm{AD}$, the search for new and potent inhibitors is of significant interest and a progressive area of current research.

Ammodaucus leucotricus belongs to the family Apiaceae, it is an endemic plant and comprises one species in Algeria (Quezel, 1963). A. leucotrichus is used by the Algerian Saharan population to treat stomach diseases, fever, vomits, allergies and is also emmenagogue, abortive and aphrodisiac (Maiza et al., 2014). Several studies have shown that $A$. leucotrichus is rich in essential oil (Gherraf et al., 2013; El-Haci et al., 2014).

Chemically, the essential oil of this species is characterized by the presence of perillaldehyde $(58.3 \%)$ and limonene $(23.33 \%)$, which were previously studied for their anticholinesterase and monoamine oxidase inhibitory activities (Sadaoui et al., 2018). Antibutyrylcholinesterase and anti-monoamine oxidase activities have been reported for perilladehyde, in contrast, limonene showed only anti-acetylcholinesterase activity (Sadaoui et al., 2018).

This work is a continuation of the research work cited above and which consists of an in-depth analysis of the interactions of the tested compounds (perilladehyde and D-limonene) with the enzymes in question. In order to have this better vision and reading of these interactions, molecular docking was carried out.

\section{Methodology}

\section{Molecular Docking Study}

Molecular docking study was conducted using Molecular Operating Environment (MOE), 2016.08 in order to explore the binding mode of the tested compound (D-Limonene) against Acetylcholinesterase (AChE) and Butyrylcholinesterase (BChE) while compound Perillaldehyde against $\mathrm{AChE}$ and Monoamine Oxidase (MAO) enzymes. The 3D structures for both compounds were generated using the MOE-builder module of MOE. Next, both the compounds were protonated and were energy minimized using the default parameters of MOE (Gradient: 0.05, Force Field: MMFF94X). The structural coordinates for AChE, $\mathrm{BChE}$ and MAO were retrieved from protein databank using PDB code 1acl, 1p0p and 4a79, respectively. All the structure was subjected to MOE for preparation. Next, all the structures were subjected to energy minimization to get the minimal energy conformation of each target. Finally, all the refined structures were used for docking purposes using the default parameters of MOE; Placement: Triangle Matcher, Rescoring-1: London dG, Refinement: Forcefield, Rescoring-2: GBVI/WSA. Before running the docking protocol, we have selected a total of ten conformations for the ligand. The top-ranked conformations based on docking scores were selected for Protein-Ligand Interaction (PLI) analysis.

\section{Results and Discussion}

Molecular docking study has been applied to elucidate the interactions occurring in ChE and MAO and their inhibitors. Molecular docking results revealed that both the compounds showed the fit-well mode of binding in the active site of the targeted enzyme. In case of D-Limonene against both the targeted enzyme (AChE and BChE) showed favorable $\mathrm{H}$-phi interaction with catalytic residue, Trp82 against AChE while with Trp84 against BChE (Fig. 1A and 1B), which might have crucial role in inhibition. Both the enzyme shared protein sequence similarity index by more $80 \%$. While in case of compound perillaldehyde against $\mathrm{BChE}$ and MAO enzyme (Fig. 1C and 1D), the docking results indicate that against $\mathrm{BChE}$ enzyme, compound perillaldehyde showed best interaction profile as compare with compound D-Limonene. The high potency and interaction profile might be due the additional attached electron-donating group (EDG), i.e., $\mathrm{OH}$ at -para position, which might activate the compound and hence raised the inhibitory potential against BChE enzyme.

Similarly, against MAO enzyme, compound perillaldehyde showed also good interaction profile, i.e., residue Lys296 and Gly58. Overall, these results delineated that perillaldehyde showed best potential for BChE enzyme might be due to the EDG whereas compound D-Limonene lack.

Perilladehyde and limonene was the main components of the essential oil of A. leucotrichus. These monoterpènes are present with a percentage of $(58.3 \%)$ and $(23.33 \%)$ respectively. The previous study showed that limonene has inhibitory activity only against AchE with an IC50 of $51.6 \mathrm{ug} / \mathrm{mL}$. While, the perillaldehyde showed inhibitory activity against $\mathrm{BuChE}$ and MAO with IC50 values of $42.7 \mathrm{ug} / \mathrm{mL}$ and $100.4 \mathrm{ug} / \mathrm{mL}$, respectively. The in-vitro results show that perilladehyde had a good inhibitory activity of the enzyme BuChE compared to the inhibition of the enzyme AchE by the limonene. These results were confirmed by the molecular docking (in silico study) where it was shown that the perilladehyde-BuChE interaction was better than the AchE-limonene interaction. The in-vitro enzymatic activity best correlates well with the in-silico molecular docking study. 


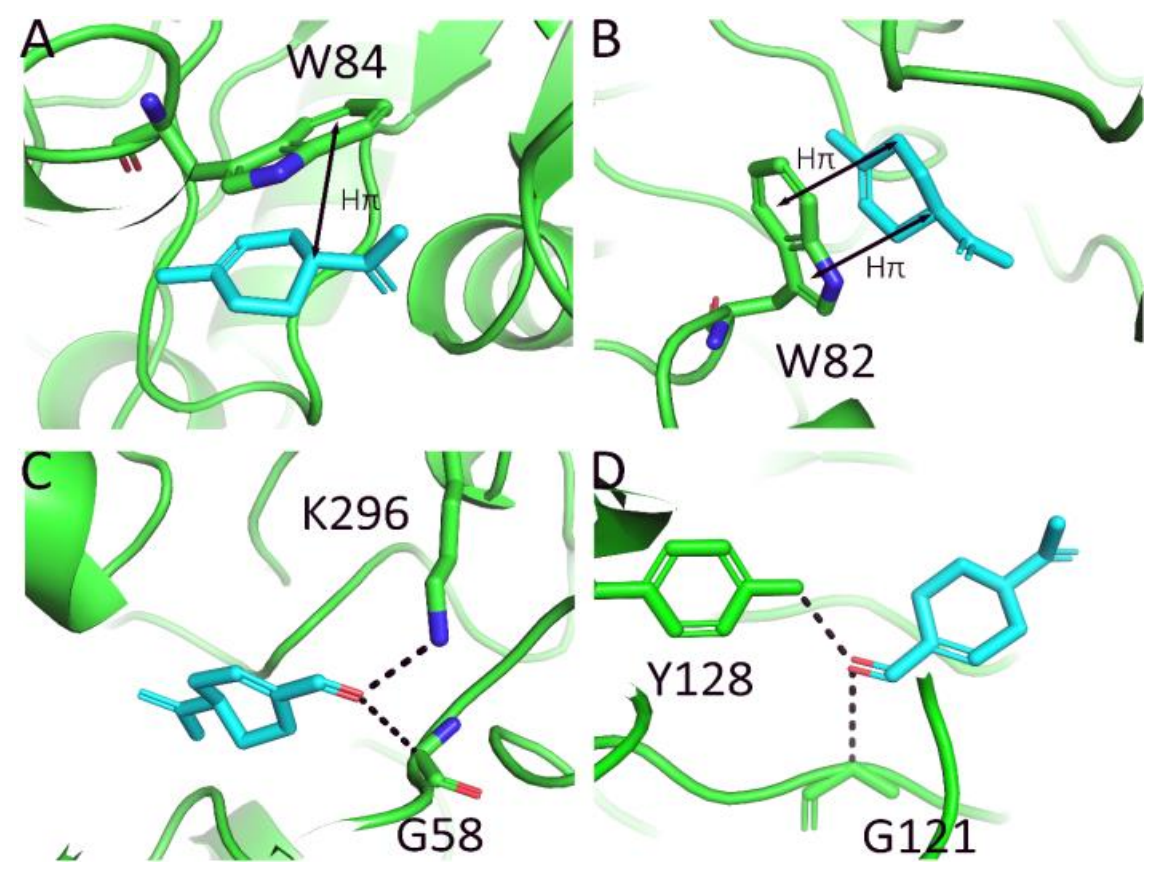

Fig. 1: The PL interaction profiles for D-limonene and perillaldehyde against AChE, BuChE and MAO enzyme. (A) represent the interaction profile for compound D-limonene against AChE; Docking Score -4.87565613 and (B) for BuChE enzyme; Docking Score -4.67808199. (C) Represent the interaction profile for compound perillaldehyde against MAO; Docking Score -5.28108454 and (D) for BuChE enzyme; Docking Score -4.85081863. D-limonene and perillaldehyde were colored into Cyan, while residues into green. Hydrogen bonding is shown in black color dotted lines

\section{Conclusion}

The molecular docking was used to determine the interactions between perilladehyde and limonene with the receptor binding-pocket of the cholinesterase (AChE and $\mathrm{BuChE}$ ) and monoamine oxidase enzymes. Based on this study, it was observed that the D-limonene showed favorable H-phi interaction with catalytic residue of enzymes and perilladehyde also showed good interaction with BChE and MAO enzymes. This work constitutes the beginning of research for a hoped-for objective of arriving at designing new drugs for the treatment of neurodegenerative diseases.

\section{Acknowledgement}

Thanks for the "Direction Générale de la Recherche Scientifique et du Développement Technologique (DGRSDT)" of Algerian Ministry of Higher Education and Scientific Research for sopporting this work by a PRFU project.

Nesrine SADAOUI acknowledges Nicole BEC and LARROCHE Christian for their contribution to elucidation of cholinesterase and monoamine oxidase inhibitory activities (determination of IC50 values). The authors thank Professor Khemili Amina for her careful reading.

\section{Author's Contributions}

Nesrine Sadaoui-Smadhi: Participated in all experiments, coordinated the data-analysis and contributed to the writing of the manuscript.

Souad Khemili-Talbi and Wafa Mokhtari: Designed the research plan (molecular docking), organized the in silico study and contributed to the writing of the manuscript.

Rahim Fazal and Wadood Abdul: Participated in moleculat docking and in the analysis of the results.

Souheyla Toubal and Narimen Benhabyles: Participated in biological activities of A. leucotrichus.

Karim Arab and Khettal Bachra: Designed the research plan (the experimental study) and organized the study.

\section{Conflicts of Interest}

The authors declare no conflict of interest.

\section{References}

Cesura, A.M. and A. Pletscher, 1992. The new generation of monoamine oxidase inhibitors. Prog. Drug Res., 38: 171-297.

DOI: 10.1007/978-3-0348-7141-9_3 
Dall'Acqua, S., F. Maggi, P. Minesso, M. Salvagno and F. Papa et al., 2010. Identification of non-alkaloid acetylcholinesterase inhibitors from Ferulago campestris (Besser) Grecescu (Apiaceae). Fitoterapia, 81: 1208-1212. DOI: $10.1016 /$ j.fitote.2010.08.003

Darvesh, S., D. Grantham and D.A. Hopkins, 1998. Distribution of butyrylcholinesterase in the human amygdala and hippocampal formation. J. Comparative Neurol., 393: 374-390. DOI: 10.1002/(SICI)10969861(19980413)393:3<374::AID-CNE8>3.0.CO;2-Z

El-Haci, I.A., C. Bekhechi, F. Atik-Bekkara, W Mazari and M. Gherib et al., 2014. Antimicrobial activity of Ammodaucus leucotrichus fruit oil from Algerian Sahara. Natural Product Commun., 9: 711-712. DOI: $10.1177 / 1934578 X 1400900533$

Gherraf, N., A. Zellagui, A. Kabouche, M. Lahouel and R. Salhi, 2013. Chemical constituents and antimicrobial activity of essential oils of Ammodaucus leucotricus. Arabian J. Chemis., 10: S2476-S2478. DOI: 10.1016/j.arabjc.2013.09.013

Lu, S.H., J.W. Wu, H.L. Liu, J.H. Zhao and K.T. Liu et al., 2011. The discovery of potential acetylcholinesterase inhibitors: A combination of pharmacophore modeling, virtual screening and molecular docking studies. J. Biomed. Sci., 18: 8-8. DOI: 10.1186/1423-0127-18-8

Maiza, A.B. and K.M.V.H. Michele Guyot, 2014. Biological activities of plants collected in the Algerian Sahara. Life Sci. Leaflets.

MOE, 2016.08. Chemical Computing Group Inc., 1010 Sherbrooke St. West, Suite \#910, Montreal, QC, Canada, H3A 2R7.

Perry, E.K., R.H. Perry, G. Blessed and B.E. Tomlinson, 1978. Changes in brain cholinesterases in senile dementia of Alzheimer type. Neuropathol. Applied Neurobiol., 4: 273-277.

DOI: $10.1111 /$ j.1365-2990.1978.tb00545.x
Quezel, P.S., 1963. Nouvelle flore de l'Algérie et des régions désertiques méridionales.

Quinn, D.M., 1987. Acetylcholinesterase: Enzyme structure, reaction dynamics and virtual transition states. Chem. Rev., 87: 955-979. DOI: $10.1021 / \mathrm{cr} 00081 \mathrm{a} 005$

Sadaoui, N., N. Bec, V. Barragan-Montero, N. Kadri and F. Cuisinier et al., 2018. The essential oil of Algerian Ammodaucus leucotrichus Coss. and Dur. and its effect on the cholinesterase and monoamine oxidase activities. Fitoterapia, 130: 1-5. DOI: 10.1016/j.fitote.2018.07.015

Sussman, J.L., M. Harel, F. Frolow, C. Oefner and A Goldman et al., 1991. Atomic structure of acetylcholinesterase from Torpedo Californica: A prototypic acetylcholine-binding protein. Science, 253: 872-879. DOI: 10.1126/science. 1678899

Terry, A.V. and J.J. Buccafusco, 2003. The cholinergic hypothesis of age and Alzheimer's disease-related cognitive deficits: recent challenges and their implications for novel drug development. J. Pharmacol. Exp. Therapeut., 306: 821-827.

DOI: $10.1124 /$ jpet.102.041616

Wright, C.I., C. Geula and M.M. Mesulam, 1993. Neuroglial cholinesterases in the normal brain and in Alzheimer's disease: relationship to plaques, tangles and patterns of selective vulnerability. Annals Neurol., 34: 373-384. DOI: 10.1002/ana.410340312

Youdim, M.B., 1995. The advent of selective monoamine oxidase A inhibitor antidepressants devoid of the cheese reaction. Acta Psychiatrica Scandinavica, 91: 5-7. DOI: 10.1111/j.1600-0447.1995.tb05917.x 